\title{
On the Motion of the Aircraft under Alternate Wing Pressure Loading*
}

\author{
Lazarus Teneketzis TENEK ${ }^{* *}$
}

\begin{abstract}
An aircraft structure is considered freely in air, unsupported. It is discretized with a set of triangular shell finite elements. It is subjected to alternate pressure forces on its wings. The governing equation is the nonlinear dynamics equation, the nonlinearity arising from the inclusion of the geometrical stiffness allowing for large displacements. The time dependent nonlinear problem is solved by a finite difference scheme. Time-displacement curves are given for a point on the tip of the wing and on the body shell. Under alternate pressure forces -as in case of turbulence- the wings displace upwards in a nonlinear way and then rigid body motion of the aircraft takes place. The motion has chaotic characteristics.
\end{abstract}

Key Words: Aircraft, Computational Model, Computational Simulation

\section{Introduction}

The aircraft is a state of the art engineering structure. In flight, the aircraft operates and sustains loads such as pressure and temperature freely and unsupported under free-free conditions. Since these loads are dynamic in nature, the aircraft vibrates and thus becomes of interest its response in time. Under turbulence, alternating and random pressure loads are imposed on the aircraft and it is of interest the initial and sequent motion of the aircraft as a whole. It is also of interest to see if the response of the structure has chaotic characteristics.

The modeling and mechanical behavior of the aircraft would not be possible without the finite element method which we shall employ here to study our structure. The aircraft structure is a thin shell structure and in this study will be modeled with triangular shell finite elements. This is a first discretization of the structure which in reality has also stiffeners which we will not take in account in this study. We mention that the problem in hand consists a large computational problem -a problem of large scale computing. An effort was undertaken here to model the structure with a reasonable amount of shell finite elements since a very fine discretization may increase significantly the time of computation. However, we mention in passing that our shell element converges rapidly, for an essential

* Received 10th December, 2004 (No. 04-5189)

** Centre for Techno-Mathematics \& Scientific Computing Laboratory, HSCS, University of Westminster, London HA1 3TP, UK.

E-mail: 1tenek@yahoo.com and meaningful simulation to be performed. Naturally, the problem studied in this work leaves open the question for a future more precise structural modeling, vibration over longer periods of time, smaller time steps and generally a finer computation.

The author undertook a study, whereby the dynamic and chaotic characteristics of a three-dimensional arc structure was studied. It was a thermally stressed space arc subjected to oscillating temperature comprising its critical temperature and a slight sinusoidal variation ${ }^{(1)}$. A dynamic nonlinear finite element problem was formulated and solved using an unconditionally stable timedependent scheme. An extensive study was undertaken to extract the chaotic characteristics that the space arc exhibited. In a second study ${ }^{(2)}$, the dynamics of the arc were studied under a mid arc vertical buckling load. The same methodology as in the first study was used. The dynamics showed again chaotic characteristics.

In this study, we build on our previous experience with structural chaotic dynamics and apply our experience to study the dynamics of a free-free aircraft structure. Isotropic triangular shell elements are used. We impose alternating -with time- pressure loads on the wings and extract the initial and subsequent response of two points on the tip of the wing and on the body shell, respectively. The nonlinear dynamics equation is here solved by a finite difference scheme. We plot the initial response of the structure, comment on the subsequent motion, display two attractors and plot the Lyapunov exponent for the acceleration of the tip of the wing. 


\section{Computational Model}

The equilibrium state of a discretized system of forces in an inertial system is described by the fundamental equation of dynamics ${ }^{(3)}$, where the inertial forces plus the damping forces plus the elastic forces are equal to the externally applied forces. In the present work we use the finite element method to model and study the response of the aircraft structure. The triangular shell element TRIC is used $^{(4),(5)}$. The element is based on the natural-mode finite element method. It has three nodes and six global Cartesian degrees of freedom per node, that is three translations and three rotations. The global degrees of freedom are so combined to yield, in the natural coordinate that follows the triangle's edges, 6 rigid-body and 12 straining modes. The straining modes are equal to the total global Cartesian degrees of freedom minus the number of the rigid body modes. Continuing, the elemental stiffness matrix is taken from Refs. (4) and (5) while the elemental mass matrix is taken from Ref. (6). By invoking standard finite element procedures, the equation of dynamics is stated

$$
M r^{\prime \prime}+C r^{\prime}+K r=R
$$

where $M$ is the global mass matrix, $C$ the damping matrix, $K$ the global stiffness matrix, and $R$ the vector of applied loads. In case of large displacements and nonlinearity, the stiffness matrix is replaced by the tangential stiffness which is the summation of the elastic and geometrical stiffnesses, namely

$$
K=K_{E}+K_{G}
$$

We solve Eq. (1) by adopting a finite difference scheme in time whereby the displacement, velocity and accelerations are expanded in Taylor's series, namely

$$
\begin{aligned}
& f(x)=f(\alpha)+\frac{f^{\prime}(\alpha)}{1 !}(x-a)+\frac{f^{\prime \prime}(\alpha)}{2 !}(x-\alpha)^{2} \\
& +\ldots . .+\frac{f^{(n-1)}(\alpha)}{(n-1) !}(x-\alpha)^{n-1}
\end{aligned}
$$

which yields

$$
\begin{aligned}
& u(t+\Delta t)=u(t)+u^{\prime}(t) \Delta t+\frac{1}{2} u^{\prime \prime}(t) \Delta t^{2} \\
& u^{\prime}(t+\Delta t)=u^{\prime}(t)+u^{\prime \prime}(t) \Delta t \\
& u^{\prime \prime}(t+\Delta t)=M^{-1}\left[R-F^{v}-F^{c}\right]
\end{aligned}
$$

\section{Computational Simulation}

Figure 1 shows a schematic of an aircraft with some basic dimensions, material parameters and the swept-back wing angle. The finite element mesh of the aircraft structure is shown in Fig. 2. The structure is discretized with 165 nodes and 214 triangular shell elements. The total number of degrees of freedom are 732. A finer discretization at this point would present presently computational expense that would call for large-scale computing. However, the triangular shell element TRIC $^{(4)}$ converges

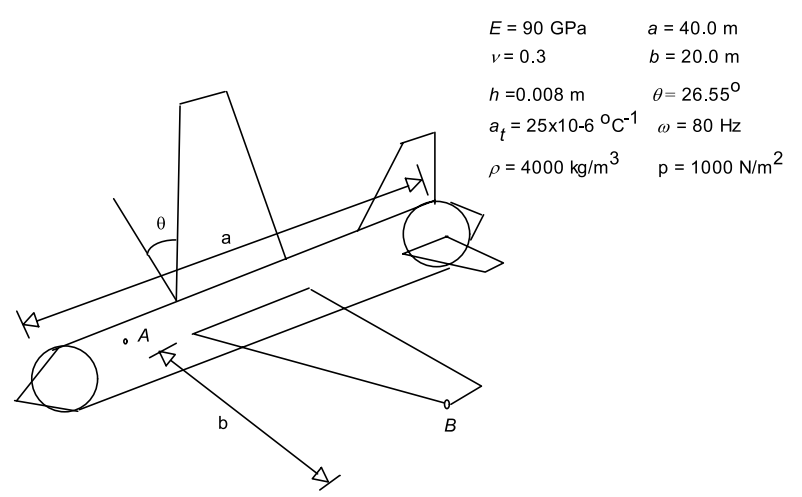

Fig. 1 A schematic of the aircraft structure

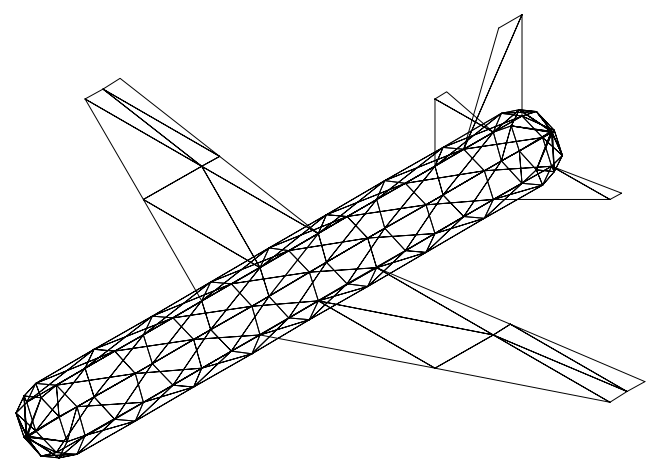

Fig. 2 Finite element mesh of the aircraft
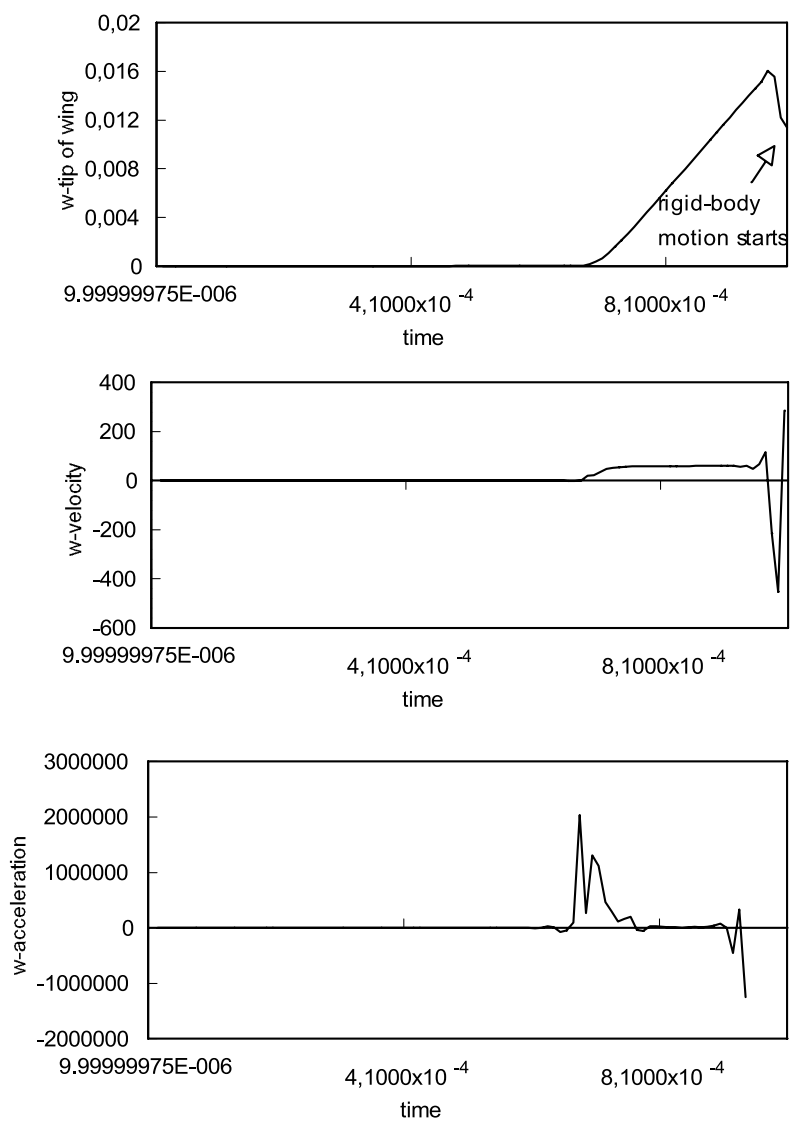

Fig. 3 Vertical time-displacement, velocity, acceleration curves for a point at the tip of the wing 

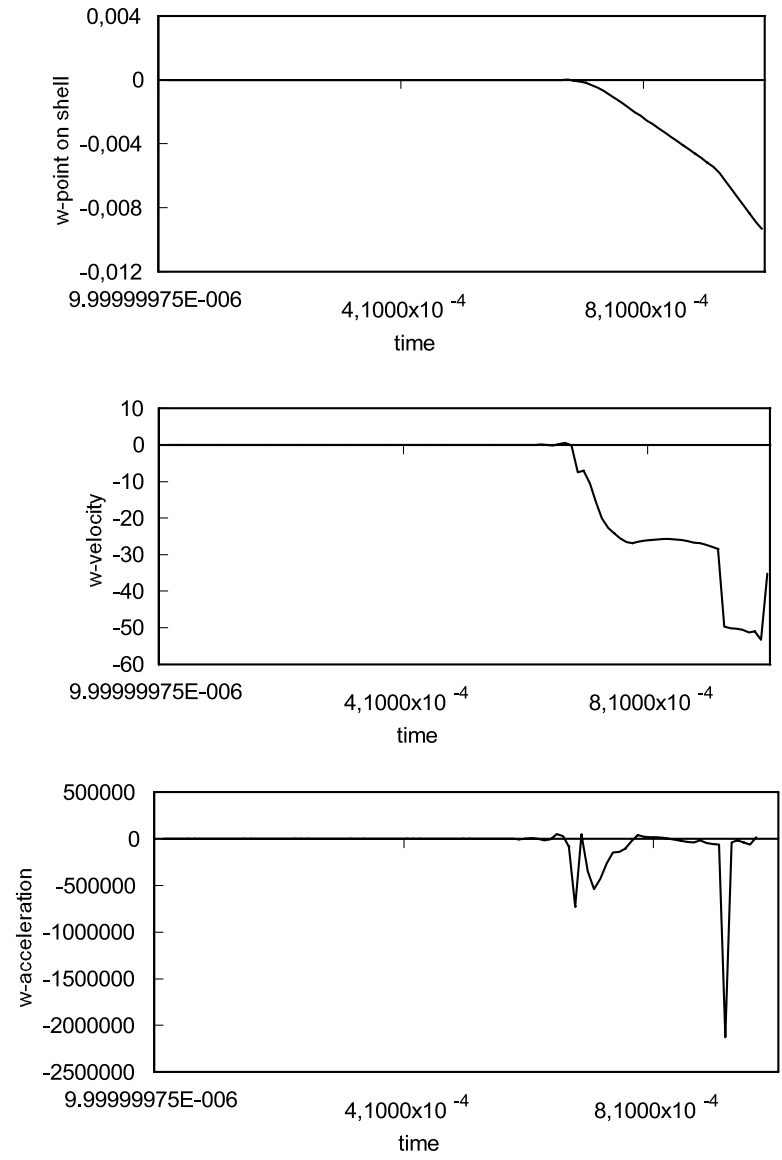

Fig. 4 Vertical time-displacement, velocity, acceleration curves for a point at the middle-top of the body shell

fast and the author asserts that with the current finite element mesh the basic dynamic characteristics of the aircraft structure can be studied. The aircraft is unsupported moving freely in space. The load is considered a normal pressure on the wings alternating with the time. The numerical algorithm was executed over 100 time steps with a time step equal to $1 \times 10^{-5}$ seconds. A small time step was necessary in order to study the dynamic response of the aircraft at the initial stage of motion. Figure 3 presents the time-displacement curves for the vertical displacement, velocity and acceleration of a point at the wing tip, and Fig. 4 presents the same curves for a point at the middle top of the body shell. From Fig. 3 we observe that the wing initially reacts little, than it starts displacing upwards and subsequently it starts displacing downwards and at this point the aircraft starts moving as a rigid body in large increments. The same motion was given by the computer for the point on the body shell. So under the considered alternate wing pressure loads the wings of the aircraft displace (tense) upwards and result to a rigid body motion of the aircraft. Velocity and acceleration time curves are also provided in Figs. 3 and 4. This rigid-body motion of the aircraft due to alternate wing loads in the finding of the current work. Figure 5 shows the attractors of the tip
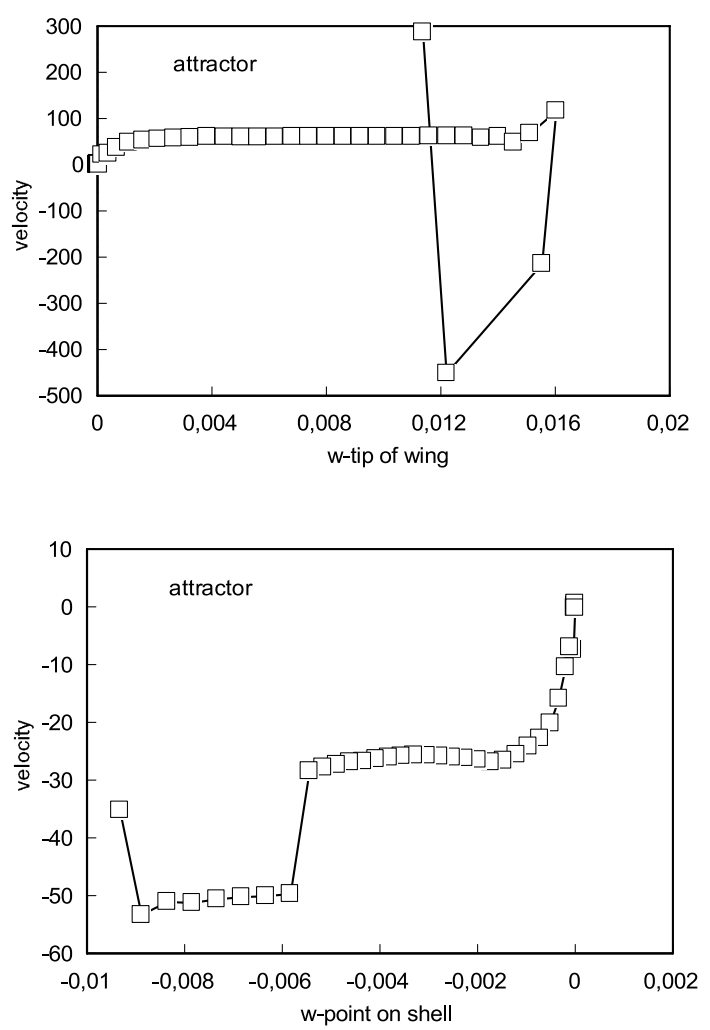

Fig. 5 Attractors

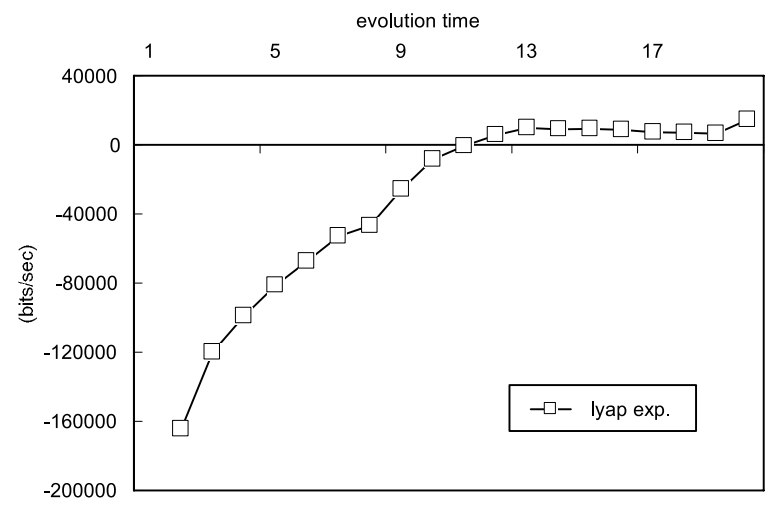

Fig. 6 Lyapunov exponent of the vertical acceleration of the wing tip

and body shell point. The attractors are in the beginning smooth and later they start to open up. Figure 6 displays the largest Lyapunov exponent of the acceleration of the point at the tip of the wing. We observe from the plot that the largest Lyapunov exponent converges positively over an evolution time. This fact asserts that the motion of the aircraft under the imposed loading inherits chaotic characteristics.

\section{Conclusions}

A model aircraft is considered freely unsupported. It is discretized with a set of triangular shell finite elements. A nonlinear dynamic problem (large displacements) is formulated with alternate -with time- pressure loads imposed 
on the wings. The aircraft structure vibrates under the imposed wing loads and we display time-displacement curves for the displacement, velocity and acceleration for a point at the tip of the wing and for a point on the body shell. The wings of the aircraft initially displace upwards, and then rigid-body motion of the aircraft starts. Attractors for the two points are plotted. A positive Lyapunov exponent for the acceleration of the tip of the wing suggests chaotic characteristics of the motion. The problem is a problem of large-scale computing.

\section{References}

( 1 ) Tenek, L.T., Chaos in a Thermally Stressed Space Arc, AIAA Journal, Vol.40, No.12 (2002), pp.2511-2516.

( 2 ) Tenek, L.T., Vibration of a Space Arc Subject to a Critical Dynamic Load, in Press, Communications in $\mathrm{Nu}$ - merical Methods in Engineering.

( 3 ) Argyris, J. and Mlejnek, H.P., Dynamics of Structures, (1991), Elsevier, North-Holland, Amsterdam.

( 4 ) Argyris, J., Tenek, L. and Olovsson, L., TRIC: A Simple but Sophisticated 3-Node Triangular Element Based on 6 Rigid-Body and 12 Straining Modes for Fast Computational Simulations of Arbitrary Isotropic and Laminated Composite Shells, Computer Methods in Applied Mechanics and Engineering, Vol.145 (1997), pp.11-85.

( 5 ) Tenek, L.T. and Argyris, J., Finite Element Analysis for Composite Structures, (1998), Kluwer Academic, Dordrecht, The Netherlands.

( 6 ) Argyris, J., Tenek, L. and Olovsson, L., Nonlinear Free Vibration of Composite Plates, Computer Methods in Applied Mechanics and Engineering, Vol.122 (1994), pp.1-51. 